\title{
Age-related differences in valence and arousal ratings of pictures from the International Affective Picture System (IAPS): Do ratings become more extreme with age?
}

\author{
DANIEL GRÜHN \\ University of Geneva, Geneva, Switzerland \\ AND \\ SuSANNe SCheibe \\ Max Planck Institute for Human Development, Berlin, Germany
}

\begin{abstract}
The International Affective Picture System (IAPS) has been widely used in aging-oriented research on emotion. However, no ratings for older adults are available. The aim of the present study was to close this gap by providing ratings of valence and arousal for 504 IAPS pictures by 53 young and 53 older adults. Both age groups rated positive pictures as less arousing, resulting in a stronger linear association between valence and arousal, than has been found in previous studies. This association was even stronger in older than in young adults. Older adults perceived negative pictures as more negative and more arousing and positive pictures as more positive and less arousing than young adults did. This might indicate a dedifferentiation of emotional processing in old age. On the basis of a picture recognition task, we also report memorability scores for individual pictures and how they relate to valence and arousal ratings. Data for all the pictures are archived at www.psychonomic.org/archive/.
\end{abstract}

The role of age in modulating emotion-cognition interactions has become a focus of increasing interest in the field of life span psychology in recent years (Blanchard-Fields, 2005; Carstensen \& Mikels, 2005). Consequently, the need for information on young and older adults' perception of the stimulus material used in this research has increased. So far, however, such age-normative information has often not been available. Previous studies on the impact of age on emotional processing have based their stimulus selection on the available normative ratings for young adults. This procedure of using data from young adults to define positive, negative, and neutral material ignores the possibility of age-related or cohort-related differences in the perception and associated meaning of the material. As a function of life experience, amount of exposure to certain situations or objects, and age-related biological changes, some stimuli may be perceived as more or less negative (or positive), or more or less arousing, by certain age groups than by others. To verify that stimuli produce similar emotional reactions across young and older age groups, previous studies have often collected valence and/or arousal ratings a posteriori. It would, however, be desirable to have rating data for older adults to guide stimulus selection during the planning phase of experimental studies. The goal of this study is to close this gap and provide valence and arousal ratings for young and older adults.
One well-known and widely used stimulus set of pictorial affective material is the International Affective Picture System (IAPS; Lang, Bradley, \& Cuthbert, 1998). The IAPS was developed to provide a large set of standardized, emotionally evocative, internationally accessible photographs from a wide range of semantic categories, including animals, people, landscapes, objects, war scenes, sexual interactions, illness, and others. Based on a dimensional approach to emotion (Osgood, Suci, \& Tannenbaum, 1957; Russell, 2003; Wundt, 1897), the IAPS provides ratings on the dimensions of valence and arousal. ${ }^{1}$ These ratings were collected mainly from psychology students (Ito, Cacioppo, \& Lang, 1998; Lang et al., 1998; Libkuman, Otani, Kern, Viger, \& Novak, 2007; Ribeiro, Pompéia, \& Bueno, 2005). The dimension of valence (i.e., pleasantness) differentiates positive (pleasant) from negative (unpleasant) emotional states. The dimension of arousal (i.e., activation) differentiates highly exciting, arousing states from calm, relaxed states. To assess the dimensions of valence and arousal, Lang and colleagues used the Self-Assessment Manikin (SAM; Lang, 1980), a nonverbal affective rating system. In this system, a series of graphic figures is presented for each dimension. They range from a smiling, happy figure to a frowning, unhappy figure for valence, and from an excited, wide-eyed figure to a relaxed, sleepy figure for arousal. Together, each series forms a 9-point rating

D. Grühn, daniel.gruehn@pse.unige.ch 
scale. Empirical data show that the valence and arousal dimensions are not independent of each other (Ito et al., 1998; Lang et al., 1998; Libkuman et al., 2007; but see Ribeiro et al., 2005). Rather, they form a U-shaped function: Unpleasant pictures tend to be more highly arousing than pleasant pictures, and both pleasant and unpleasant pictures are typically more arousing than neutral pictures.

Can valence and arousal ratings of young adults be generalized to older age groups? The literature on emotion and aging indicates that younger and older adults differ in several aspects of emotional functioning. For example, cross-sectional (e.g., Mroczek \& Kolarz, 1998) and longitudinal (e.g., Kunzmann, Little, \& Smith, 2000) studies suggest that, in everyday life, older adults experience negative affect less frequently than younger adults do and positive affect about as frequently. Older adults report decreased surgency (the tendency to experience positive emotions very intensely), greater stability of mood, and decreased sensation seeking (Lawton, Kleban, Rajagopal, $\&$ Dean, 1992). They further report having better developed emotion regulation capacities (Carstensen, Pasupathi, Mayr, \& Nesselroade, 2000; Gross et al., 1997). In addition, there is some evidence that older adults show reduced autonomic reactions to emotional stimuli (e.g., Levenson, Carstensen, Friesen, \& Ekman, 1991; but see Kunzmann \& Grühn, 2005). These age-related differences in emotional experience, control, and reactivity may affect the perceived valence and arousal of emotional pictures. For example, older adults' reduced autonomic reactivity to emotional stimuli may lead them to perceive emotional pictures as less arousing.

A few previous studies have obtained valence and/or arousal ratings of IAPS pictures from young and older adults, with inconsistent results (Backs, da Silva, \& Han, 2005; Charles, Mather, \& Carstensen, 2003; Mather \& Knight, 2005; Mikels, Larkin, Reuter-Lorenz, \& Carstensen, 2005; Smith, Hillman, \& Duley, 2005; Wieser, Mühlberger, Kenntner-Mabiala, \& Pauli, 2006). Most of these studies have used only a small subset of IAPS pictures and, sometimes, oversimplified ratings, which has restricted the possibility of obtaining significant age-related differences in the perception of affective pictures. Charles et al. used 27 IAPS pictures in their study of emotional memory. They asked young and older participants to categorize pictures as positive, neutral, or negative. The authors stated that the subjective valence categorizations mapped the a priori valence categories. However, the assessment of the subjective categorizations was probably too coarse to show age-related shifts in stimuli connotation. Mikels et al. also found no age differences when they asked young and older participants to rate the emotional intensity of 40 neutral, 40 positive, and 40 negative IAPS image pairs. Age differences were also absent in the valence and arousal ratings of 41 IAPS images used in Study 2 by Mather and Knight and in the valence and arousal ratings of 54 IAPS images used by Wieser and colleagues.

Other studies, however, have reported age differences in IAPS ratings between young and older adults. In their Study 1, Mather and Knight (2005) found that overall, older adults rated a set of 41 IAPS images more positively than young adults did. Specifically, older adults rated neutral and positive pictures more positively than young adults did, whereas the two groups did not differ in their valence ratings for negative pictures. Smith et al. (2005) investigated self-reports of valence and arousal in response to 45 IAPS pictures that were selected to depict, equally often, scenes of younger adults, scenes of older adults, or scenes that were not age associated. Older adults rated the pictures as more positive and more arousing than young adults did. Unfortunately, the authors did not investigate whether the age relevance of the pictures had any influence on these ratings. Backs et al. (2005) conducted the most systematic study on age differences in affective experiences of IAPS pictures. They collected valence and arousal ratings of 90 IAPS pictures from 21 young and 21 older adults. The pictures were selected to equally represent five different regions of the evaluative space formed by valence and arousal: pleasant-aroused, pleasant-calm, neutral, unpleasant-calm, and unpleasant-aroused. Age group differences emerged in the quadratic relationship between valence and arousal, which was less pronounced in older than in young adults. A closer look revealed, however, that these age differences were restricted to pleasant pictures: Young adults rated pleasant-aroused pictures as more arousing and pleasant than older adults did, whereas older adults rated pleasant-calm pictures as more pleasant, yet less arousing, than young adults did. The differential ratings by young and older adults may reflect an age-related increase in a preference for low-arousing stimuli and activities. This seems to be consistent with the above-mentioned finding of lower surgency and sensation seeking in older adults (Lawton et al., 1992).

Thus, some studies have shown age differences in the perception of the IAPS pictures, whereas others have not. Most studies have used, however, only a small subset of IAPS pictures, which has limited their ability to find age differences and to generalize to other pictures. Moreover, most studies have not provided ratings for individual pictures. The goal of the present study was to investigate the impact of age on valence and arousal ratings of IAPS pictures and to make these ratings available to other researchers. To provide a useful set for picture selection in future studies, we expanded past research by investigating a larger set of pictures. Young and older adults completed a recognition memory task involving 504 IAPS pictures and subsequently rated the pictures for valence and arousal, using the SAM figures (Lang, 1980). We report valence and arousal data for each of the 504 IAPS pictures separately for younger and older adults and correlate these ratings with past ratings (Ito et al., 1998; Lang et al., 1998; Libkuman et al., 2007; Ribeiro et al., 2005). We also report younger and older adults' memorability scores for each picture on a recognition task.

\section{METHOD}

\section{Participants}

Fifty-three young (18-31 years of age, $M=25.23, S D=3.39$; $45.3 \%$ of them female) and 53 older adults (63-77 years of age, $M=$ 
Table 1

Descriptive Statistics for the 504 IAPS Pictures and for the Subsets of 168 Negative, 168 Neutral, and 168 Positive Pictures Based on Normative Ratings (Lang, Bradley, \& Cuthbert, 1998)

\begin{tabular}{|c|c|c|c|c|c|c|c|c|}
\hline \multirow[b]{2}{*}{ Rating Source } & \multicolumn{2}{|c|}{ Total } & \multicolumn{2}{|c|}{ Negative } & \multicolumn{2}{|c|}{ Neutral } & \multicolumn{2}{|c|}{ Positive } \\
\hline & $M$ & $S D$ & $M$ & $S D$ & $M$ & $S D$ & $M$ & $S D$ \\
\hline \multicolumn{9}{|c|}{ Valence Ratings } \\
\hline Young adults & 4.93 & 1.59 & 3.01 & 0.72 & 5.32 & 0.73 & 6.44 & 0.67 \\
\hline Older adults & 5.02 & 1.94 & 2.70 & 0.96 & 5.55 & 0.88 & 6.82 & 0.84 \\
\hline Lang, Bradley, \& Cuthbert, 1998 & 5.05 & 1.86 & 2.81 & 0.59 & 5.15 & 0.43 & 7.20 & 0.47 \\
\hline Ito, Cacioppo, \& Lang, 1998 & 5.17 & 2.00 & 2.84 & 0.73 & 5.08 & 0.69 & 7.14 & 0.74 \\
\hline Libkuman, Otani, Kern, Viger, \& Novak, 2007 & 4.27 & 1.80 & 2.25 & 0.72 & 4.31 & 0.76 & 6.10 & 1.01 \\
\hline Ribeiro, Pompéia, \& Bueno, 2005 & 5.16 & 2.38 & 2.27 & 0.83 & 5.28 & 0.90 & 7.70 & 0.65 \\
\hline \multicolumn{9}{|c|}{ Arousal Ratings } \\
\hline Young adults & 4.12 & 1.54 & 5.98 & 0.94 & 3.18 & 0.78 & 3.20 & 0.66 \\
\hline Older adults & 4.31 & 1.93 & 6.71 & 1.09 & 3.31 & 0.84 & 2.90 & 0.74 \\
\hline Lang et al., 1998 & 4.73 & 1.16 & 5.39 & 0.76 & 3.53 & 0.83 & 5.27 & 0.76 \\
\hline Ito et al., 1998 & 4.81 & 1.31 & 5.64 & 1.06 & 3.45 & 0.95 & 4.92 & 1.01 \\
\hline Libkuman et al., 2007 & 3.59 & 1.07 & 4.00 & 0.85 & 2.66 & 0.84 & 4.04 & 0.90 \\
\hline Ribeiro et al., 2005 & 5.40 & 1.62 & 7.25 & 0.91 & 4.61 & 0.80 & 4.42 & 1.18 \\
\hline
\end{tabular}

Note-Descriptive statistics for Ito et al. (1998), Libkuman et al. (2007), and Ribeiro et al. (2005) are based on subsets of 290 pictures ( 99 negative, 70 neutral, and 121 positive), 430 pictures (141 negative, 136 neutral, and 153 positive), and 426 pictures (139 negative, 135 neutral, and 152 positive), respectively.

69.61, $S D=3.58 ; 52.8 \%$ of them female) were recruited in Berlin for two testing sessions via local newspaper advertisements. The sample was stratified by educational level $(54.7 \%$ with 12 and more years of schooling; $45.3 \%$ with less than 12 years of schooling). The participants received $€ 30$ as compensation.

Due to the stratification procedure, young $(M=13.56, S D=3.39)$ and older $(M=13.17, S D=4.09)$ adults were similar regarding years of education $\left[F(1,104)=0.28, p=.60, \eta^{2}<.01\right]$. As has been generally found in past research, young adults $(M=56.8, S D=$ 11.3) performed better than older adults $(M=41.5, S D=8.9)$ in a perceptual speed task (digit symbol substitution task; Wechsler, 1981) $\left[F(1,104)=59.52, p<.01, \eta^{2}=.36\right]$, whereas older adults $(M=35.47, S D=3.71)$ were better than young adults $(M=32.54$, $S D=4.27$ ) in a vocabulary task (WST; Schmidt \& Metzler, 1992) $\left[F(1,104)=14.15, p<.01, \eta^{2}=.12\right]$. Age groups did not differ in positive affect [young, $M=4.65, S D=1.05$; old, $M=5.01, S D=$ $\left.0.89 ; F(1,101)=3.62, p=.06, \eta^{2}=.03\right]$, measured with the PANAS (Watson, Clark, \& Tellegen, 1988), using a scale from 1 (not at all, hardly) to 7 (very strongly, extremely). However, young adults reported more negative affect $(M=3.85, S D=0.94)$ than older adults $\operatorname{did}(M=2.80, S D=0.77)\left[F(1,101)=38.76, p<.01, \eta^{2}=.28\right]$

\section{Stimuli}

For this study, 504 pictures were selected from the IAPS (Lang et al., 1998), representing an equal number of negative $(n=168)$, neutral $(n=168)$, and positive $(n=168)$ pictures. We excluded pictures that (1) revealed major sex-related differences in valence and arousal ratings from the IAPS normative data, (2) were ambiguous in the depicted content, or (3) were of poor photographic quality. On the basis of the normative data by Lang and colleagues, positive and negative pictures were selected to match on emotional arousa $\left[F(1,334)=2.19, p=.14, \eta^{2}<.01\right]$. However, neutral pictures in the selected set revealed lower arousal ratings than did negative $\left[F(1,334)=463.24, p<.01, \eta^{2}=.58\right]$ and positive $[F(1,334)=$ 404.46, $p<.01, \eta^{2}=.55$ ] pictures. Table 1 provides mean IAPS normative ratings of valence and arousal for the selected pictures. Figure 1A shows the distribution of the 504 selected pictures based on the normative ratings provided by Lang and colleagues.

Two research assistants coded the IAPS pictures for their content - that is, whether they displayed predominantly people, animals, scenery/landscapes, or objects (rater agreement: $\kappa=.93$ ). Our selected set of pictures was composed of 277 people, 151 object, 47 animal, and 29 landscape pictures.

\section{Procedure}

At the beginning of the first session, the participants performed a picture recognition task (for approximately $20 \mathrm{~min}$; for details of the recognition task, see Grühn, Scheibe, \& Baltes, 2007) and two cognitive tasks $(\sim 15 \mathrm{~min})$ : a perceptual speed task and a vocabulary task. After these tasks, the participants were asked to rate 252 pictures on valence and arousal. Thus, each participant rated only one half of the total picture set. To do this, the 504 pictures were divided into four sets with an equal number of positive, negative, and neutral pictures. Each participant received two sets; sets were counterbalanced across participants. In a first block, all 252 pictures were rated on valence; in a second block, the same 252 pictures were rated on arousal. The pictures were presented on a computer screen at random with the corresponding 9-point SAM figure below. To complete the rating task, the participants took 20-35 min. The second session (approximately 1 week after the first session) was devoted primarily to the assessment of person characteristics (including trait affect).

\section{RESULTS}

\section{Correlations Between Valence and Arousal in Present and Previous Ratings}

To verify the generalizability of the obtained ratings, we compared our ratings of young and older adults with available ratings of young adults from previous studies (Ito et al., 1998; Lang et al., 1998; Libkuman et al., 2007; Ribeiro et al., 2005). ${ }^{2}$ Whereas the normative data by Lang and colleagues provide ratings for all 504 pictures, the sets used by Ito et al., Libkuman et al., and Ribeiro et al. overlapped with the present set of pictures for only 290 pictures (99 negative, 70 neutral, and 121 positive; categorization based on the normative ratings by Lang et al., 1998), 430 pictures (141 negative, 136 neutral, and 153 positive), and 426 pictures (139 negative, 135 neutral, and 152 positive), respectively. Table 2 provides the intercorrelation matrix: Valence ratings in the present study were highly consistent with previous valence ratings $(.85 \leq r \leq$ $.92)$, suggesting that the participants in this study agreed substantially with those in previous studies on which pictures they perceived as positive, neutral, and negative. In 
A

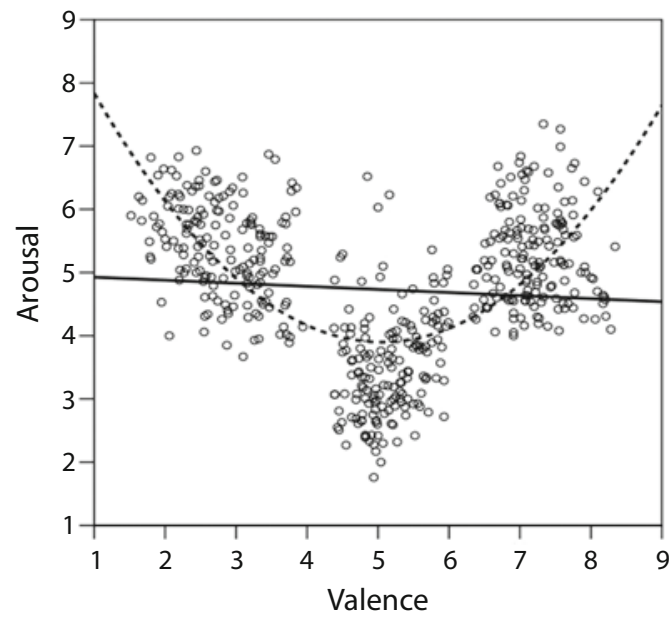

C

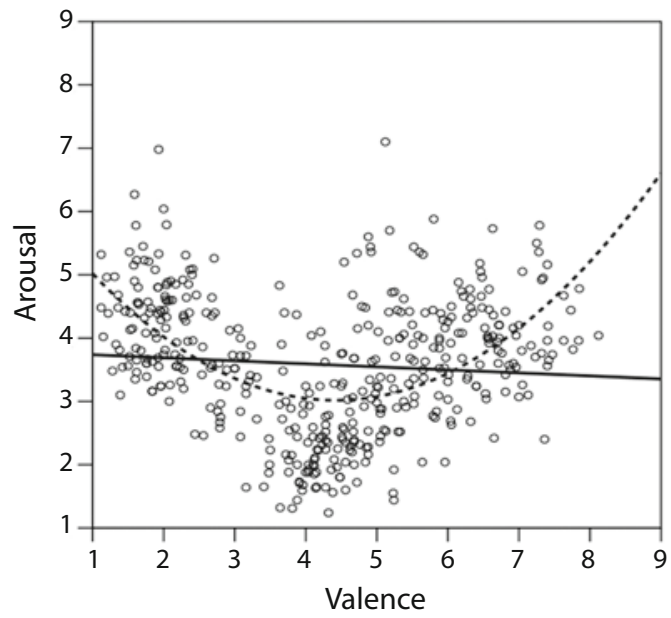

E

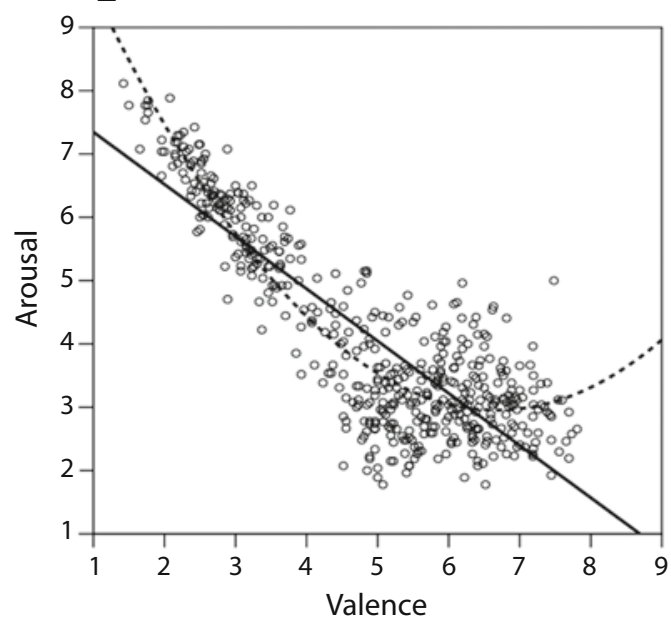

B

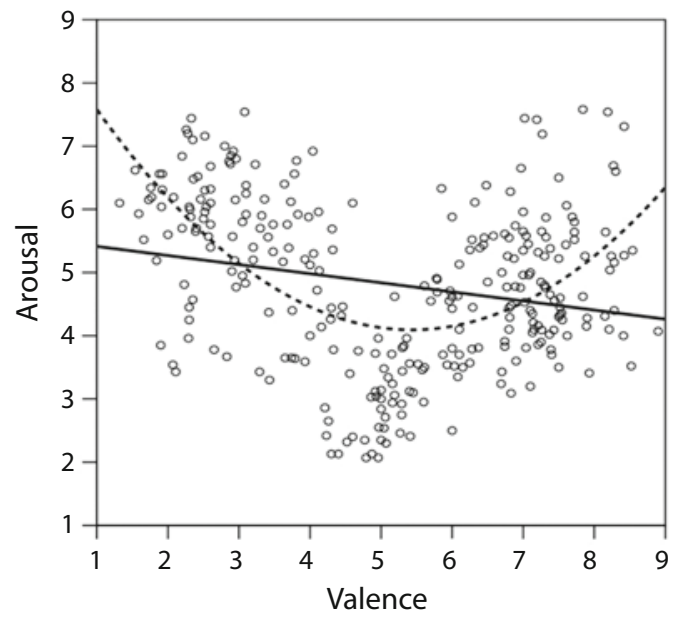

D

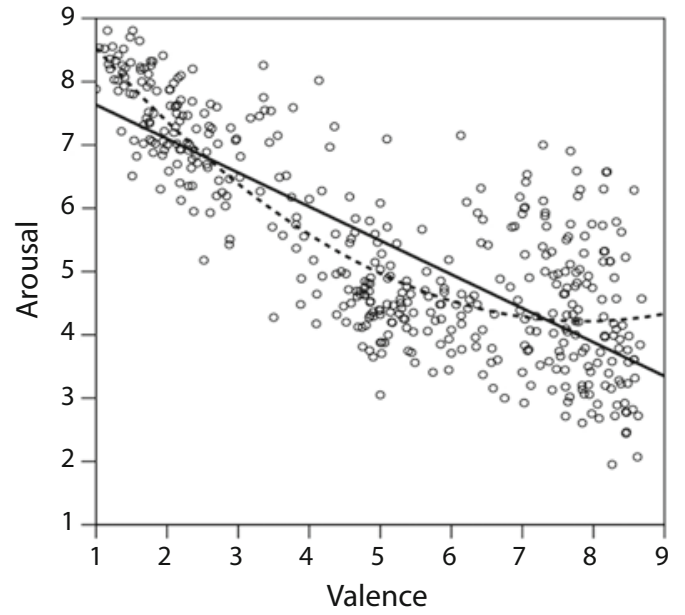

$\mathbf{F}$

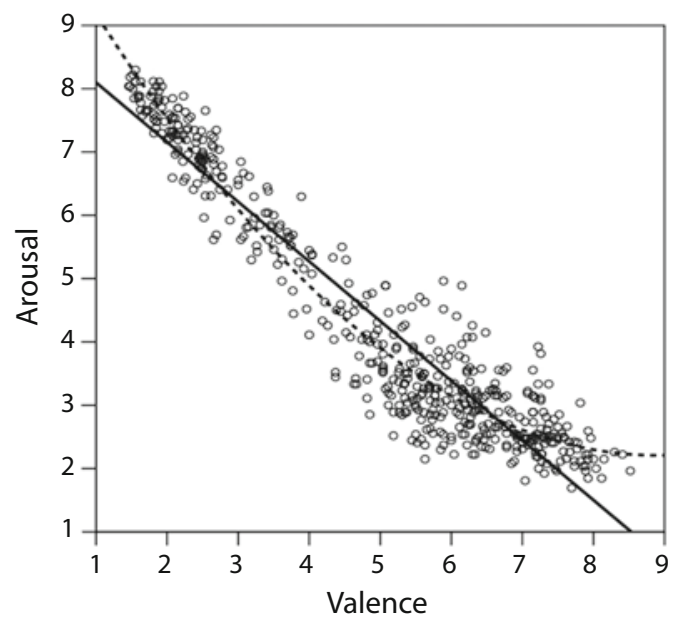

Figure 1. Scatterplots for valence and arousal ratings of the 504 pictures selected from the International Affective Picture System by different rating sources: (A) Lang, Bradley, and Cuthbert, 1998; (B) Ito, Cacioppo, and Lang, 1998 (only 290 pictures); (C) Libkuman, Otani, Kern, Viger, and Novak, 2007 (only 430 pictures); (D) Ribeiro, Pompéia, and Bueno, 2005 (only 426 pictures); (E) present study's young adults' ratings; and (F) present study's older adults' ratings. The linear and quadratic associations between valence and arousal ratings are represented by solid and dashed lines, respectively. 
Table 2

Correlation Matrix of Valence and Arousal Ratings by Young and Older Adults in the Present Study and From Past Rating Studies for the 504 IAPS Pictures

\begin{tabular}{|c|c|c|c|c|c|c|c|c|c|c|c|}
\hline & 1 & 2 & 3 & 4 & 5 & 6 & 7 & 8 & 9 & 10 & 11 \\
\hline \multicolumn{12}{|c|}{ Valence Ratings } \\
\hline \multicolumn{12}{|l|}{ 1. Young adults } \\
\hline 2. Old adults & $.95^{* *}$ & & & & & & & & & & \\
\hline 3. Lang, Bradley, \& Cuthbert, 1998 & $.92^{* *}$ & $.90^{* *}$ & & & & & & & & & \\
\hline 4. Ito, Cacioppo, \& Lang, 1998 & $.91^{* *}$ & $.89^{* *}$ & $.96^{* *}$ & & & & & & & & \\
\hline \multicolumn{12}{|l|}{ 5. Libkuman, Otani, Kern, Viger, } \\
\hline 6. Ribeiro, Pompéia, \& Bueno, 2005 & $.93^{* *}$ & $.92^{* *}$ & $.96^{* *}$ & .00 & $.89^{* *}$ & & & & & & \\
\hline \multicolumn{12}{|c|}{ Arousal Ratings } \\
\hline 7. Young adults & $-.85^{* *}$ & $-.86^{* *}$ & $-.77^{* *}$ & $-.79^{* *}$ & $-.75^{* *}$ & $-.79^{* *}$ & & & & & \\
\hline 8. Old adults & $-.91^{* *}$ & $-.95^{* *}$ & $-.84^{* *}$ & $-.83^{* *}$ & $-.81^{* *}$ & $-.87^{* *}$ & $.93^{* *}$ & & & & \\
\hline 9. Lang et al., 1998 & $-.23^{* *}$ & $-.27^{* *}$ & -.08 & $-.11^{*}$ & $-.11^{*}$ & -.08 & $.55^{* *}$ & $.46^{* *}$ & & & \\
\hline 10. Ito et al., 1998 & $-.36^{* *}$ & $-.39^{* *}$ & $-.22^{* *}$ & $-.22^{* *}$ & $-.24^{* *}$ & $-.27^{* *}$ & $.61^{* *}$ & $.53^{* *}$ & $.80^{* *}$ & & \\
\hline 11. Libkuman et al., 2007 & -.09 & $-.12^{*}$ & .01 & -.01 & -.07 & .00 & $.35^{* *}$ & $.27^{* *}$ & $.70^{* *}$ & $.56^{* *}$ & \\
\hline 12. Ribeiro et al., 2005 & $-.80^{* *}$ & $-.83^{* *}$ & $-.75^{* *}$ & $-.77^{* *}$ & $-.73^{* *}$ & $-.79^{* *}$ & $.86^{* *}$ & $.88^{* *}$ & $.54^{* *}$ & $.62^{* *}$ & $.31^{* *}$ \\
\hline
\end{tabular}

Note-Correlations with ratings by Ito et al. (1998), Libkuman et al. (2007), and Ribeiro et al. (2005) are based on overlapping subsets of 290, 430 , and 426 pictures, respectively. ${ }^{*} p<.05 .{ }^{* *} p<.01$.

contrast, arousal ratings were only moderately correlated with previous arousal ratings $(.26 \leq r \leq .88)$. Thus, the present participants agreed considerably but also deviated significantly from participants' ratings in previous studies in terms of which pictures they perceived as low, intermediately, and highly arousing. However, arousal ratings were highly correlated with previous valence ratings $(-.75 \leq r \leq-.84)$. Moreover, both dimensions, valence and arousal, were highly intercorrelated within subsamples of young $(r=-.85)$ and older $(r=-.95)$ adults. ${ }^{3}$ This suggests that the participants had a strong tendency to rate negative pictures as more arousing than positive pictures. In other words, the participants in this study did not seem to differentiate much between arousal and valence.

The magnitude of the association between valence and arousal was surprising, since it deviated from the pattern reported in previous studies (Backs et al., 2005; Ito et al., 1998; Lang et al., 1998; Libkuman et al., 2007; but see Ribeiro et al., 2005). These studies showed a U-shaped function between arousal and valence: Very positive and very negative pictures were typically rated as highly arousing, whereas less emotional and neutral pictures were rated as less arousing. Indeed, we selected our pictures on the basis of such a U-shaped association: Looking at the normative ratings for the selected 504 pictures by Lang and colleagues, there was no significant linear association $\left(R^{2}<\right.$ $.01)$, but there was a significant quadratic association $\left(R^{2}=.39\right)$ between valence and arousal (see Figure 1A). In our ratings for the same set of pictures, however, there was a strong linear association $\left(R^{2}=.86\right)$ and only a weak, although significant, quadratic association $\left(R^{2}=.05\right)$. Generally, our participants rated positive pictures as less arousing than did previous participants.

Regarding the impact of age, young and older adults' ratings revealed a highly similar correlation pattern. This was also indicated by high between-age-group correlations for valence $(r=.95)$ and arousal $(r=.93)$. However, older adults showed an even stronger association between valence and arousal $(r=-.95)$ than young adults $\operatorname{did}(r=-.85)$
$(Z=-2.88, p<.01)$. Examining linear and quadratic associations between valence and arousal within each age group further supported this finding. Older adults' valence ratings had a stronger linear association with arousal $\left(R^{2}=\right.$ $.90)$ than young adults' ratings did $\left(R^{2}=.73\right)$. In contrast, ratings of valence showed a stronger quadratic association with arousal for young adults $\left(R^{2}=.10\right)$ than with that for older adults $\left(R^{2}=.03\right.$; all four $\left.p \mathrm{~s}<.01\right)$. Figure 1 presents individual pictures rated by young (panel E) and older (panel \#F) adults, together with estimated lines for linear (solid) and quadratic (dashed) effects.

\section{Mean Differences in Valence and Arousal in Present and Previous Ratings}

Table 1 provides mean ratings of valence and arousal from the present and previous rating studies (Ito et al., 1998; Lang et al., 1998; Libkuman et al., 2007; Ribeiro et al., 2005 ) across the 504 IAPS pictures and separately for positive, negative, or neutral pictures classified on the basis of the normative IAPS ratings. ${ }^{4}$ In a first step, we compared ratings by young and older adults. In a second step, we compared our ratings with the normative IAPS ratings. To compare valence and arousal ratings across age groups, two $3 \times 2$ (valence category $\times$ age group) mixed ANOVAs were performed at the picture level. Valence category (negative, neutral, or positive, based on normative ratings) served as the between-pictures factor, and age group (young or old) as the within-pictures factor. For both dimensions, we obtained significant main effects of age group [valence, $F(1,501)=$ $16.88, \eta^{2}=.03$; arousal, $\left.F(1,501)=43.88, \eta^{2}=.08\right]$ and valence category [valence, $F(2,501)=1,120.27, \eta^{2}=.82$; arousal, $\left.F(2,501)=912.51, \eta^{2}=.79\right]$, as well as significant interactions between age group and valence category [valence, $F(2,501)=62.99, \eta^{2}=.20$; arousal, $F(2,501)=$ $108.68, \eta^{2}=.30 ;$ all $\left.p s<.01\right]$.

Follow-up analyses for each valence category revealed a general pattern that older adults' ratings were more extreme than ratings by young adults. Regarding valence, older adults rated negative pictures as more negative than 

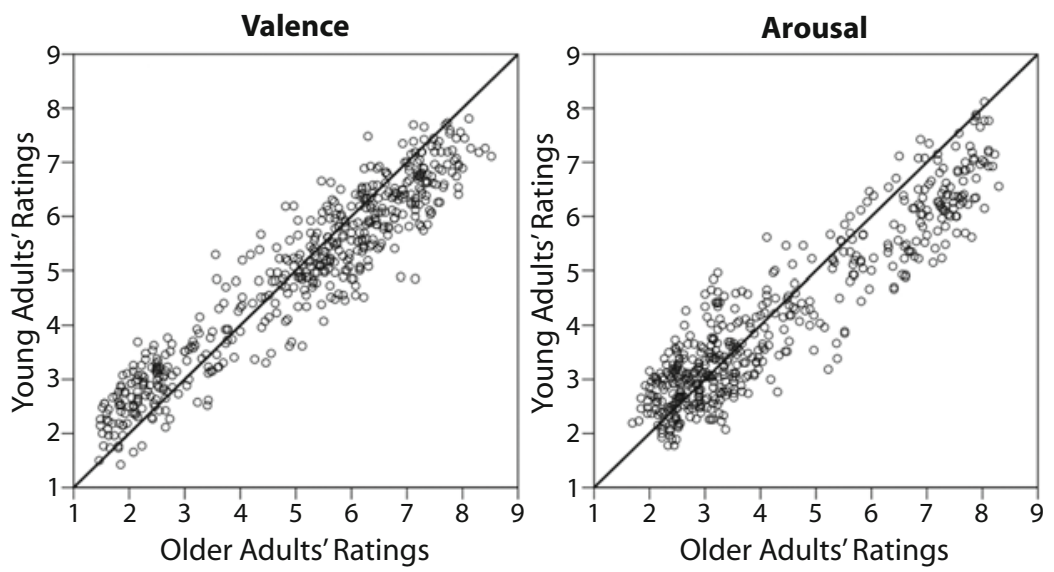

Figure 2. Scatterplots between young and older adults' ratings for the 504 pictures from the International Affective Picture System, separately for valence and arousal. The solid line represents perfect fit $(r=1.00)$.

young adults did $\left[F(1,167)=45.89, \eta^{2}=.22\right]$. In contrast, older adults rated neutral $\left[F(1,167)=29.12, \eta^{2}=\right.$ $.15]$ and positive $\left[F(1,167)=65.59, \eta^{2}=.28\right]$ pictures as more positive than young adults did. Regarding arousal, older adults rated negative $\left[F(1,167)=178.46, \eta^{2}=\right.$ $.52]$ and neutral $\left[F(1,167)=8.72, \eta^{2}=.05\right]$ pictures as more arousing than young adults did, whereas they rated positive pictures as less arousing than young adults did $\left[F(1,167)=38.69, \eta^{2}=.19\right.$; all $p$ s $\left.<.01\right]$. Thus, older adults rated negative pictures as more negative and more arousing and positive pictures as more positive and less arousing than young adults did.

To contrast our ratings with the normative ratings (Lang et al., 1998), we conducted separate univariate ANOVAs for each valence category, age group, and rating dimension. For valence, our obtained ratings showed a tendency to the neutral midpoint of the response scale: As compared with the normative ratings, positive pictures were rated as less positive [young, $F(1,167)=277.53, p<.01, \eta^{2}=.62$; old, $\left.F(1,167)=36.55, p<.01, \eta^{2}=.18\right]$, neutral pictures were rated as somewhat more positive [young, $F(1,167)=10.76$, $p<.01, \eta^{2}=.06$; old, $F(1,167)=45.61, p<.01, \eta^{2}=$ $.22]$, and negative pictures were rated as less negative by young adults $\left[F(1,167)=18.36, p<.01, \eta^{2}=.10\right]$ and similarly negative by older adults $[F(1,167)=18.36, p=.08$, $\left.\eta^{2}=.02\right]$. In contrast, arousal ratings by young and older adults were more extreme (i.e., biased toward the endpoints of the response scale) than the normative ratings: Positive [young, $F(1,167)=2,561.81, \eta^{2}=.94$; old, $F(1,167)=$ $\left.2,034.42, \eta^{2}=.92\right]$ and neutral [young, $F(1,167)=30.76$, $\eta^{2}=.16$; old, $\left.F(1,167)=8.39, \eta^{2}=.05\right]$ pictures were rated as less arousing, whereas negative pictures were rated as more arousing [young, $F(1,167)=85.59, \eta^{2}=.34$; old, $F(1,167)=338.93, \eta^{2}=.67$; all $p$ s $\left.<.01\right]$.

\section{Age-Related Differences in Valence and Arousal Ratings of Individual Pictures}

To address the question of age-related differences in the perception of individual pictures, univariate ANOVAs were conducted for each picture. Age (young vs. old) was treated as a between-subjects factor, and the respective valence or arousal rating as a dependent variable. This procedure resulted in 2 (dimensions) $\times 504$ (pictures) $=$ 1,008 univariate analyses. The large number of analyses was expected to result in several significant effects by chance (approximately 5\% significant results for an alpha level of .05). Nevertheless, the significance level was not adjusted, since we considered it more important to maximize our chance of identifying all pictures with agerelated differences than to minimize the risk of false alarms. The archived file contains these picturewise analyses for valence and arousal ratings. ${ }^{5}$

Figure 2 presents scatterplots between young and older adults' ratings separately for valence and arousal; each dot represents 1 of the 504 pictures. Although the correlation between valence ratings for young and older adults was extremely high $(r=.95)$, the analyses for valence revealed a substantial number of significant mean differences at the level of individual pictures: The valence ratings for young and older adults differed for 151 pictures $(30.0 \%$ of 504 pictures): Young adults rated 89 pictures $(17.7 \%)$ as more positive (or less negative) and 62 pictures $(12.3 \%)$ as more negative (or less positive) than older adults did. As is evident in Figure 2, older adults rated positive pictures more positively and negative pictures more negatively than young adults did. The analyses for arousal also revealed a substantial number of significant mean differences by age group. Specifically, arousal ratings between young and older adults differed for 94 pictures (18.7\%): Older adults rated 73 pictures as more arousing and 21 pictures as less arousing than young adults did. In general, older adults rated negative pictures as more arousing and positive pictures as less arousing than young adults did.

Which pictures revealed significant age differences? In an exploratory approach, we looked at the specific content of these pictures. The IAPS pictures cover a wide and diverse range of scenes and themes. Pictures with age dif- 
ferences in valence or arousal ratings were found in every content area. However, we observed that a large proportion of pictures with age differences, in contrast to ageinvariant pictures, were found in two domains: pictures of couples and pictures of violent acts. In general, older adults rated pictures of couples more positively and pictures of violence more negatively than young adults did. ${ }^{6}$

Taken together, the number of significant age-related differences in both valence and arousal ratings of individual pictures was much higher than was to be expected by chance. Most age differences were found in valence ratings. To the extent that these age differences in picture perception are related to memory for or attention to these pictures, this finding has substantial implications for aging-oriented research with IAPS pictures. In other words, observed age differences in processing emotional pictures may be partly explained by age differences in the perception of these pictures.

\section{Follow-Up Analyses on the Memorability of Individual Pictures}

To provide an initial impression on how age might alter the relation between perception (i.e., ratings of valence and arousal) and processing of emotional pictures, we examined memorability scores for each picture - that is, whether certain pictures are better remembered than others. Our data set provides the opportunity to report such memorability scores. In the recognition task, each picture appeared equally often as a target and a distractor item. Therefore, we were able to compute for each picture a discrimination index $d^{\prime}$ that indicates how easily a picture was discriminated as a target or as a distractor item (Snodgrass \& Corwin, 1988). These memorability scores are also provided in the online supplement.

Table 3 shows correlations between memorability and ratings of valence and arousal across the 504 pictures. Ratings of valence were negatively correlated with memorability in young adults, signifying that young adults recognized negative pictures better than they did positive pictures. There was, however, no significant association between ratings of valence and memorability in older adults. This pattern was evident across rating sources (present and past ratings). The correlation between arousal and memorability depended on the source of the arousal rating. Present ratings of arousal were positively related to memorability in young adults, whereas normative arousal ratings were negatively related to memorability in older adults. However, after partialing out valence, memorability by young adults was unrelated to arousal, whereas memorability by older adults was now consistently negatively correlated with arousal ratings.

\section{DISCUSSION}

The goal of the present study was to provide valence and arousal ratings of young and older adults for 504 IAPS pictures (Lang et al., 1998). In the social-cognitive aging literature, IAPS pictures are widely used, for example, in experiments on emotion-cognition interactions (e.g., Charles et al., 2003; Mather \& Knight, 2005; Mikels et al., 2005). However, the frequently used procedure of using ratings of young adults to classify stimulus material for young and older adults does not take potential age-related or cohort-related shifts in the perception of the material into account. To address this issue, the present article reports mean and correlational differences between valence and arousal ratings for young and older adults and makes these ratings available to other researchers.

The present study revealed three major findings: First, both age groups rated positive pictures as less arousing than was to be expected from the normative IAPS ratings. This pattern brought about a heightened linear association (and weaker quadratic association) between valence and arousal ratings. Second, the linear association between valence and arousal was even stronger (and the quadratic association was even weaker) for older than for young adults. Finally, there were substantial age-related differences in the perception of individual pictures, especially for valence. In general, older adults' evaluations were more extreme in that positive pictures were rated more positively and negative pictures more negatively than was the case for young adults. Similarly, older adults rated positive pictures as less arousing and negative pictures as more arousing than young adults did.

\section{Consistencies and Inconsistencies Between Present and Previous Ratings}

Comparing the present ratings with previous ones, the pattern was clear-cut. For valence, ratings for young and older adults were highly correlated with previous ratings.

Table 3 Correlations Between Memorability and Ratings of Valence and Arousal for Young and Older Adults

\begin{tabular}{|c|c|c|c|c|c|c|c|c|}
\hline \multirow[b]{3}{*}{ Rating Source } & \multicolumn{8}{|c|}{ Correlation Between Memorability and } \\
\hline & \multicolumn{4}{|c|}{ Valence for } & \multicolumn{4}{|c|}{ Arousal for } \\
\hline & \multicolumn{2}{|c|}{ Young Adults } & \multicolumn{2}{|c|}{ Older Adults } & \multicolumn{2}{|c|}{ Young Adults } & \multicolumn{2}{|c|}{ Older Adults } \\
\hline Young adults & $-.16^{*}$ & $(-.07)$ & .03 & $(-.08)$ & $.15^{*}$ & $(.02)$ & -.08 & $(-.11)^{*}$ \\
\hline Older adults & $-.16^{*}$ & $(-.08)$ & .03 & $(-.14)^{*}$ & $.14^{*}$ & $(-.03)$ & -.08 & $(-.16)^{*}$ \\
\hline Lang, Bradley, \& Cuthbert, 1998 & $-.15^{*}$ & $(-.15)^{*}$ & .00 & $(-.02)$ & .00 & $(-.02)$ & $-.18^{*}$ & $(-.18)^{*}$ \\
\hline Ito, Cacioppo, \& Lang, 1998 & $-.12^{*}$ & $(-.13)^{*}$ & .05 & $(.02)$ & .00 & $(-.04)$ & $-.18^{*}$ & $(-.18)^{*}$ \\
\hline Libkuman, Otani, Kern, Viger, \& Novak, 2007 & $-.13^{*}$ & $(-.13)^{*}$ & .01 & $(.00)$ & .01 & $(.00)$ & $-.10^{*}$ & $(-.10)^{*}$ \\
\hline Ribeiro, Pompéia, \& Bueno, 2005 & $-.14^{*}$ & $(-.09)$ & .03 & $(-.12)^{*}$ & $.10^{*}$ & $(-.01)$ & $-.14^{*}$ & $(-.18)^{*}$ \\
\hline
\end{tabular}

Note-Values in parentheses are partial correlations for which the corresponding rating of arousal or valence was partialed out. Correlations for Ito et al. (1998), Libkuman et al. (2007), and Ribeiro et al. (2005) are based on 290, 430, and 426 pictures, respectively. ${ }^{*} p<.05$. 
Moreover, valence ratings for young and older adults were highly correlated with each other, suggesting a general consensus among age groups in evaluating which picture is more negative or more positive. In contrast, arousal ratings from the present study showed only modest correlations with previous arousal ratings. Notably, though, both age groups largely agreed on the rank order of pictures on the arousal dimension $(r=.93)$. A closer look revealed that the discrepancy with previous arousal ratings was especially pronounced for positive pictures: Both age groups rated positive pictures as less arousing than had been shown in previous studies.

Why did our participants rate positive pictures as low arousing rather than as highly arousing, as was also reflected in the strong linear association between valence and arousal ratings? We offer four potential explanations for these differences between present and past ratings: The different pattern may have been due to differences in historical time periods, assessment methods, habituation effects, and/or cross-cultural differences. First, the normative ratings by Lang et al. (1998) were mostly collected 10 or 20 years ago. What seems arousing may have changed over time, especially if one considers the radical change of media usage over the past few decades. For example, some pictures may clearly look as if they were taken in the 1980s. Although we tried to eliminate old-fashioned pictures from the present picture set, we were not able to exclude all of them, due to our aim to include a large subset of the IAPS in the study.

Second, the assessment methods have changed over time. The normative IAPS ratings were obtained primarily in group sessions with university students, in which pictures were projected on a wall. We, in contrast, presented pictures on a computer screen (with a SAM figure below each picture) to a more heterogeneous sample of well- and less-educated participants.

Third, we employed more pictures in one rating session than previous studies had. Habituation effects may come in with an increasing number of ratings. For example, Codispoti, Ferrari, and Bradley (2006) reported habituation effects in affective perception after 20,40, and 60 repetitions of the same IAPS pictures, in that emotional pictures were evaluated as less arousing with increasing repetitions. In the study by Codispoti and colleagues, however, both negative and positive pictures revealed habituation effects, rather than only positive pictures.

Finally, there may be cross-cultural differences in how arousing positive information is perceived to be. The present ratings were collected in a German sample, whereas previous ratings were collected mainly in American samples. Unfortunately, little is known about cross-cultural differences in picture perception. One cross-cultural study in Germany and the United States with 60 IAPS pictures (Hamm \& Vaitl, 1989) revealed a pattern similar to that in the present study: Whereas the university students in Germany showed a strong correlation between valence and arousal ratings $(r=-.79)$, the U.S. students revealed a lower association $(r=-.47)$. In this study, the U.S. sample evaluated pictures, especially erotic ones, as more positive and more arousing than the German sample did. Similarly, a Brazilian study investigated the affective experience for 707 IAPS pictures with a large sample of Brazilian university students $(N=1,062$; Ribeiro et al., 2005). The Brazilian students revealed a much higher linear association between valence and arousal $(r=-.82)$ than the normative American sample did $(r=-.25)$ for the same set of pictures. Again, this was due mainly to the tendency of Brazilians to rate positive pictures as less arousing. Moreover, the Brazilian ratings of arousal correlated much more highly with young and older adults' arousal ratings (young, $r=.86$; old, $r=.88$ ) than with the U.S. arousal ratings $(.26 \leq r \leq .61)$. Germans and Brazilians seem to perceive positive pictures differently than U.S. Americans do. Perhaps the construct of arousal is meaningfully differentiated from valence only by U.S. psychology students, rather than by samples that are more heterogeneous in terms of cultural and educational background.

\section{Older Adults Tended to Give More Extreme Valence and Arousal Ratings}

Despite the high consensus with respect to the rank order of emotional pictures, as evidenced by the high correlations between young and older adults' valence and arousal ratings, a major number of pictures revealed agerelated mean differences. Consistent with previous studies (Mather \& Knight, 2005; Smith et al., 2005), older adults rated neutral and positive pictures as more positive than young adults did. Going beyond previous studies, we also found that older adults rated negative pictures as more negative than young adults did. A similar pattern was evident for arousal: Older adults rated negative pictures as more arousing and positive pictures as less arousing than young adults did. This suggests that the older adults had a tendency to respond more extremely to affective pictures. This tendency was also evident in the analyses of individual pictures. In particular, young and older adults revealed significant mean level differences in valence and arousal for $30.0 \%$ and $18.7 \%$, respectively, of the 504 pictures. Differences were mainly in the direction that older adults gave more extreme responses (away from the midpoint of the scale).

These results largely map onto findings obtained in previous age-comparative rating studies. On the one hand, the high consensus between age groups in rank ordering pictures suggests that it might be difficult to observe age-related differences in picture perception when the set of pictures is small or the rating procedure is too coarse (Charles et al., 2003; Mather \& Knight, 2005; Mikels et al., 2005). On the other hand, when age differences in picture ratings were observed in previous studies, they were consistent mainly with the present pattern of findings. Consistent with Mather and Knight, older adults in the present study rated neutral and positive pictures more positively. However, negative pictures were also rated more negatively by our older adults, whereas Mather and Knight did not find age differences for negative pictures. A pattern similar to that found by Smith et al. (2005) emerged in our study - namely, that overall, older adults rated pictures as more positive and more arousing than young adults did. 
However, our obtained effect was moderated by a strong interaction with valence category. Finally, consistent with Backs et al. (2005), the relationship between valence and arousal was less quadratic and more linear for older adults than for young adults.

One possible explanation for the less pronounced differentiation between valence and arousal in older adults might be a dedifferentiation of the emotional space with age. In the domain of intellectual functioning, life span researchers have already provided theoretical and empirical evidence for a dedifferentiation (i.e., increase in covariation) of intellectual abilities in old age (e.g., Baltes, Lindenberger, \& Staudinger, 2006; Li et al., 2004). There is some initial evidence for a similar age-related pattern in emotional functioning (Labouvie-Vief \& Marquez, 2004). For example, Labouvie-Vief, DeVoe, and Bulka (1989) examined age differences in emotional understanding (i.e., the differentiated way in which people talk about emotional events). Levels of emotional understanding increase continuously from adolescence to midlife but decline thereafter. However, more research is necessary to uncover life span changes in the structure of emotion and emotional processing.

\section{Memorability Scores for Individual Pictures}

Our follow-up analyses on the relation of valence and arousal to memorability indicate that age differences in affective picture perception need to be taken into account when memory for emotional pictures is studied. Consistent with the literature on age-related memory for emotional information (e.g., Charles et al., 2003; Grühn et al., 2007), the more negative a picture, the better it was remembered by young adults, whereas there was no memory advantage for negative pictures in older adults. Our study suggests that this pattern might, in part, be due to the fact that older adults perceive negative pictures as more arousing. We found that arousal is somewhat beneficial to young adults' memory, although the effect disappeared after partialing out valence. In contrast, arousal appears to be harmful to older adults' memory, especially after partialing out valence. Thus, older adults may have difficulties remembering negative pictures mainly because of their highly arousing nature.

\section{Summary and Conclusion}

The present study revealed consistencies with previous rating studies of the IAPS and provides information about age-related differences in affective picture ratings. Whereas ratings of valence were highly consistent with previous ratings and across young and older adults, both age groups rated positive pictures as substantially less arousing than was found in previous studies. Consequently, valence and arousal ratings were more strongly correlated in this study than in previous studies and formed a linear, rather than a U-shaped, relationship. This linear association was especially pronounced in older adults, which was due to the older adults' tendency to rate positive pictures as much less arousing than the young adults did. Overall, older adults tended to give more extreme valence and arousal ratings to the pictures.
To conclude, our data provide a useful source for selecting age-matched IAPS pictures for future aging-oriented research. Although a substantial number of pictures showed differences between young and older adults' valence and arousal ratings, many pictures did not. From the set of 504 pictures, approximately two thirds revealed no age-related differences. Thus, there is a large pool of ageinvariant images to draw from. This will allow researchers to select pictures that are matched on valence and arousal across young and older adults for future studies.

\section{AUTHOR NOTE}

We are grateful to Paul B. Baltes for initiating and supporting this study and to Corinna Fink, Nicole Haag, Anna Katharina Ludwig, Christine Ortbandt, Maja Wiest, and Peter Paul Zurek for their assistance in recruitment and data collection. Correspondence concerning this article should be addressed to D. Grühn, FPSE-Psychology, University of Geneva, Uni-Mail, 1211 Geneva 4, Switzerland, or to S. Scheibe, Department of Psychology, Stanford University, Stanford, CA 94305 (e-mail: daniel.gruehn@pse.unige.ch or scheibe@stanford.edu).

\section{REFERENCES}

Backs, R. W., DA Silva, S. P., \& Han, K. (2005). A comparison of younger and older adults' Self-Assessment Manikin ratings of affective pictures. Experimental Aging Research, 31, 421-440.

Baltes, P. B., Lindenberger, U., \& Staudinger, U. M. (2006). Lifespan theory in developmental psychology. In W. Damon \& R. M. Lerner (Eds.), Handbook of child psychology: Vol. 1. Theoretical models of human development (6th ed., pp. 569-664). Hoboken, NJ: Wiley.

BLANCHARD-FIELDS, F. (2005). Introduction to the special section on emotion-cognition interactions and the aging mind. Psychology \& Aging, 20, 539-541.

Carstensen, L. L., \& Mikels, J. A. (2005). At the intersection of emotion and cognition: Aging and the positivity effect. Current Directions in Psychological Science, 14, 117-121.

Carstensen, L. L., Pasupathi, M., Mayr, U., \& Nesselroade, J. R. (2000). Emotional experience in everyday life across the adult life span. Journal of Personality \& Social Psychology, 79, 644-655.

Charles, S. T., Mather, M., \& Carstensen, L. L. (2003). Aging and emotional memory: The forgettable nature of negative images for older adults. Journal of Experimental Psychology: General, 132, 310-324.

Codispoti, M., Ferrari, V., \& Bradley, M. M. (2006). Repetitive picture processing: Autonomic and cortical correlates. Brain Research, 1068, 213-220.

Gross, J. J., Carstensen, L. L., Pasupathi, M., Tsai, J., Skorpen, C. G., \& Hsu, A. Y. C. (1997). Emotion and aging: Experience, expression, and control. Psychology \& Aging, 12, 590-599.

GrüHN, D., Scheibe, S., \& Baltes, P. B. (2007). Reduced negativity effect in older adults' memory for emotional pictures: The heterogeneityhomogeneity list paradigm. Psychology \& Aging, 22, 644-649.

Hamm, A. O., \& VAITL, D. (1989). Multidimensionale Analyse affektiver visueller Stimuli: Eine transkulturelle Untersuchung [Multidimensional analysis of affective visual stimuli: A cross-cultural study]. Psychologische Beiträge, 31, 125-143.

Ito, T. A., CACIOPpo, J. T., \& LANG, P. J. (1998). Eliciting affect using the International Affective Picture System: Trajectories through evaluative space. Personality \& Social Psychology Bulletin, 24, 855-879.

KunZmann, U., \& GRÜHN, D. (2005). Age differences in emotional reactivity: The sample case of sadness. Psychology \& Aging, 20, 47-59.

Kunzmann, U., Little, T. D., \& Smith, J. (2000). Is age-related stability of subjective well-being a paradox? Cross-sectional and longitudinal evidence from the Berlin Aging Study. Psychology \& Aging, 15, 511-526.

Labouvie-Vief, G., DeVoe, M., \& Bulka, D. (1989). Speaking about feelings: Conceptions of emotion across the life span. Psychology \& Aging, 4, 425-437. 
Labouvie-Vief, G., \& MarqueZ, M. G. (2004). Dynamic integration Affect optimization and differentiation in development. In D. Y. Dai \& R. J. Sternberg (Eds.), Motivation, emotion, and cognition: Integrative perspectives on intellectual functioning and development (pp. 237-272). Mahwah, NJ: Erlbaum.

LANG, P. J. (1980). Behavioral treatment and bio-behavioral assessment: Computer applications. In J. B. Sidowski, J. H. Johnson, \& T. A Williams (Eds.), Technology in mental health care delivery systems (pp. 119-137). Norwood, NJ: Ablex.

LANG, P. J., Bradley, M. M., \& CUTHBert, B. N. (1998). International affective pictures system (IAPS): Digitized photographs, instruction manual and affective ratings (Tech. Rep. A-6). Gainesville: University of Florida, NIMH Center for the Study of Emotion and Attention.

Lawton, M. P., Kleban, M. H., Rajagopal, D., \& Dean, J. (1992). Dimensions of affective experience in three age groups. Psychology \& Aging, 7, 171-184.

Levenson, R. W., Carstensen, L. L., Friesen, W. V., \& Ekman, P. (1991). Emotion, physiology, and expression in old age. Psychology \& Aging, 6, 28-35.

Li, S.-C., Lindenberger, U., Hommel, B., Aschersleben, G., Prinz, W., \& Baltes, P. B. (2004). Transformations in the couplings among intellectual abilities and constituent cognitive processes across the life span. Psychological Science, 15, 155-163.

Libkuman, T. M., Otani, H., Kern, R., Viger, S. G., \& Novak, N. (2007). Multidimensional normative ratings for the International Affective Picture System. Behavior Research Methods, 39, 326-334.

Mather, M., \& KNight, M. (2005). Goal-directed memory: The role of cognitive control in older adults' emotional memory. Psychology \& Aging, 20, 554-570.

Mikels, J. A., Larkin, G. R., Reuter-Lorenz, P. A., \& Carstensen, L. L. (2005). Divergent trajectories in the aging mind: Changes in working memory for affective versus visual information with age. Psychology \& Aging, 20, 542-553.

MroczeK, D. K., \& Kolarz, C. M. (1998). The effect of age on positive and negative affect: A developmental perspective on happiness. Journal of Personality \& Social Psychology, 75, 1333-1349.

Osgood, C. E., SucI, G. J., \& TANnenbaum, P. H. (1957). The measurement of meaning. Urbana: University of Illinois Press.

Ribeiro, R. L., Pompéia, S., \& Bueno, O. F. A. (2005). Comparison of Brazilian and American norms for the International Affective Picture System (IAPS). Revista Brasileira de Psiquiatria, 27, 208-215.

Russell, J. A. (2003). Core affect and the psychological construction of emotion. Psychological Review, 110, 145-172.

Schmidt, K.-H., \& MetZler, P. (1992). Wortschatztest-WST [Vocabulary test]. Weinheim, Germany: Beltz.

Smith, D. P., Hillman, C. H., \& Duley, A. R. (2005). Influences of age on emotional reactivity during picture processing. Journals of Gerontology, 60B, P49-P56.

SNodgrass, J. G., \& CoRwin, J. (1988). Pragmatics of measuring recognition memory: Applications to dementia and amnesia. Journal of Experimental Psychology: General, 117, 34-50.

Watson, D., Clark, L. A., \& Tellegen, A. (1988). Development and validation of brief measures of positive and negative affect: The PANAS scales. Journal of Personality \& Social Psychology, 54, 1063-1070.

WECHSLER, D. (1981). Manual for the Wechsler Adult Intelligence ScaleRevised (WAIS-R). New York: Psychological Corporation.

Wieser, M. J., Mühlberger, A., Kenntner-Mabiala, R., \& Pauli, P. (2006). Is emotion processing affected by advancing age? An eventrelated brain potential study. Brain Research, 1096, 138-147.

WUNDT, W. (1897). Outlines of psychology (C. H. Judd, Trans.). Oxford: Engelman.

\section{NOTES}

1. The IAPS also provides ratings on a third dimension, dominance (potency), ranging from a feeling of dominance, control, and self- assurance to submission, passivity, and timidity. This dimension is often less homogeneous than valence and arousal and explains only a small proportion of variance. Therefore, this dimension was not considered in this study.

2. We considered ratings from previous studies only when there was a substantial overlap in pictures used $(>100)$. Many previous rating studies used only a small set of pictures.

3. In a pilot study with 5 young and 5 older adults, we used a verbal instruction, rather than SAM figures. The scales ranged from very negative, unpleasant (1) to very positive, pleasant (9) for valence, and from calm, relaxed (1) to excited, tense (9) for arousal. In this pilot study, we also found a strong correlation between valence and arousal ratings $(r=.96)$. We initially thought that this high correlation might be due to the verbal instruction. To avoid this problem, we changed the response format to the SAM format for the normative ratings (Lang et al., 1998). The fact that we found a similarly high correlation between valence and arousal ratings with the SAM figures precludes the possibility that the high correlation was due to differences in the response format.

4. It is important to emphasize that the classification of whether a picture was considered as negative $(M \leq 4)$, neutral $(4<M \leq 6)$, or positive $(M>6)$ was based on the normative ratings by Lang et al. (1998). This was done to compare present ratings with the widely used normative ratings. However, on the basis of our present ratings, some pictures would fall in a different valence category.

5. The archived file contains picturewise analyses comparing young and older adults' ratings, but also women's and men's ratings. In contrast to age differences, the present study did not reveal a major influence of sex on the perception of the IAPS pictures. The number of sex-related differences was in the range of differences to be expected by chance, signifying that for the most part, men and women agreed on the emotional meaning of the pictorial material. Note, however, that in the present study, we had a priori excluded pictures (e.g., erotic scenes) that revealed major sex-related differences on the basis of the normative IAPS ratings (Lang et al., 1998).

6. We also explored whether the distribution of people, animal, object, and landscape pictures differed between age-invariant pictures and pictures with age differences in valence or arousal. The distribution differed only for valence ratings $\left[\chi^{2}(3, N=504)=11.89, p<.01\right]$, but not for arousal ratings $\left[\chi^{2}(3, N=504)=3.17, p=.37\right]$. The 151 pictures (30.0\% of all 504 pictures), which revealed age differences in valence represent 98 people ( $35.4 \%$ of all 277 people pictures), 36 object (23.8\% of 151$), 14$ animal (29.8\% of 47 ), and 3 landscape (10.3\% of 29$)$ pictures. Thus, most of the differences in valence ratings between age groups were found for people pictures.

\section{ARCHIVED MATERIALS}

The following materials may be accessed through the Psychonomic Society's Norms, Stimuli, and Data archive, www.psychonomic.org/ archive.

To access these files, search the archive for this article using the journal name (Behavior Research Methods), the first author's name (Grühn), and the publication year (2008).

FILE: Gruhn-BRM-2008.zip

DESCRIPTION: The compressed archive file contains two files:

Gruhn_PictureData.txt, containing IAPS picture norms for young and older adults developed by Grühn and Scheibe (2008) as a tab-delimited text file.

Gruhn_ReadMe.txt, containing a brief description of variables in the data file (Gruhn_PictureData.txt).

AUTHOR's E-MAIL ADDRESS: daniel.gruehn@pse.unige.ch

(Manuscript received June 14, 2007; revision accepted for publication August 27, 2007.) 